\title{
A Comparative Study of Three Types Shear Mode Piezoelectric Wafers in Shear Horizontal Wave Generation and Reception
}

\author{
Qiang Huan ${ }^{1,2}$, Mingtong Chen ${ }^{1}$ and Faxin $\mathrm{Li}^{1,2,3, * \mathbb{D}}$ \\ 1 LTCS and College of Engineering, Peking University, Beijing 100871, China; qiang_huan@pku.edu.cn (Q.H.); \\ chenmingtong@pku.edu.cn (M.C.) \\ 2 Center for Applied Physics and Technology, Peking University, Beijing 100871, China \\ 3 Beijing Key Laboratory of Magnetoelectric Materials and Devices, Peking University, Beijing 100871, China \\ * Correspondence: lifaxin@pku.edu.cn
}

Received: 3 July 2018; Accepted: 13 August 2018; Published: 15 August 2018

\begin{abstract}
Guided wave-based inspection has emerged as a promising tool to evaluate the reliability of key components in modern industries. The fundamental shear horizontal $\left(\mathrm{SH}_{0}\right)$ wave is always of great interests for plate-like structures because of its non-dispersion characteristics. However, the generation and reception of $\mathrm{SH}_{0}$ wave using piezoelectric wafers is not straightforward. In this paper, we firstly define three types shear mode piezoelectric wafers, i.e., the conventional in-plane poled thickness-shear $\left(d_{15}\right)$ mode, the thickness-poled thickness-shear $\left(d_{15}\right)$ mode, and the face-shear $\left(d_{24}\right)$ mode. Then, finite element simulations were conducted to demonstrate their performance in SH wave generation and reception. The results indicated that the face shear $\mathrm{d}_{24}$ wafer can generate almost single mode $\mathrm{SH}_{0}$ wave, while both types of $\mathrm{d}_{15}$ wafers would generate Lamb waves and $\mathrm{SH}_{0}$ wave simultaneously. Finally, experiments were carried out to check the efficiency of different shear mode piezoelectric wafers in $\mathrm{SH}_{0}$ wave generation and reception. The results indicated that the $\mathrm{d}_{24}$ wafer can generate and receive $\mathrm{SH}_{0}$ wave of high signal to noise ratio (SNR) with high energy conversion efficiency, while the in-plane poled $\mathrm{d}_{15}$ wafer would generate $\mathrm{SH}_{0}$ wave of high amplitude and acceptable SNR but with relatively low energy conversion efficiency. The performances of thickness-poled $\mathrm{d}_{15}$ wafer was not as good as the other two in both $\mathrm{SH}$ wave generation and reception. This work will be helpful for the applications of SH waves in plate-like structures.
\end{abstract}

Keywords: guided wave; shear horizontal wave; piezoelectric transducers; thickness shear; face shear

\section{Introduction}

Nondestructive testing (NDT) and structural health monitoring (SHM) are of great importance in modern industries to improve the reliability of structures and components [1,2]. Guided wavebased techniques have attracted special attention for their rapid and wide range of inspection applications [3-5]. For plate-like structures, there exist two types of guided waves: Lamb waves and shear horizontal (SH) waves. In the past two decades, Lamb wave-based inspections had been extensively studied and applied due to the easy excitation and reception by thickness-poled piezoelectric (PZT) wafers [6-14]. However, the inherent multi-modal and dispersive characteristics of Lamb waves have seriously restricted their further development.

In comparison, the fundamental shear horizontal $\left(\mathrm{SH}_{0}\right)$ wave in plates is completely non-dispersive. Because of its uncoupled displacements with Lamb waves, in theory it is possible to generate single mode $\mathrm{SH}_{0}$ wave. Besides, it exhibits some other attractive feathers such as less mode conversion and low attenuation in fluid-loaded plates [15-17]. Unfortunately, the generation 
and reception of $\mathrm{SH}_{0}$ wave is not as convenient as that of Lamb waves. Although in the late 1970s, Thompson had successfully excited $\mathrm{SH}_{0}$ wave in metal plates by using electromagnetic acoustic transducers (EMATs) [18,19], SH wave-based inspection has not been widely used yet because of the rather low efficiency of EMATs. Actually, $\mathrm{SH}$ waves can be excited by using the conventional in-plane poled thickness-shear $\left(\mathrm{d}_{15}\right)$ piezoelectric wafers [20]. However, Lamb waves will be excited simultaneously [21]. The conventional $\mathrm{d}_{15}$ wafers can also be assembled to be a ring or a planar array to act as the fundamental torsional $(\mathrm{T}(0,1))$ wave transducers in pipes [22-24] or omnidirectional SH wave transducers in plates [25]. Recently, another two types of shear mode piezoelectric wafers have been developed in our group for generation/reception of $\mathrm{SH}$ waves, i.e., the face-shear $\mathrm{d}_{24}$ wafer and the thickness-poled $d_{15}$ wafer [26-28]. The $d_{24}$ wafer can excite single-mode $\mathrm{SH}$ wave and receive $\mathrm{SH}$ wave only by filtering Lamb waves [26]. It can also form a ring to generate single-mode $T(0,1)[29]$ wave in pipes and form a circular array to generate $\mathrm{SH}$ wave omni-directionally in plates [30]. The advantage of the thickness-poled $d_{15}$ wafer is that it can easily form a uniform-sensitivity omni-directional $\mathrm{SH}$ wave transducer based on the thickness-poled PZT ring [27,28].

Now we can see that all the above mentioned three types of shear mode piezoelectric wafers can be used to generate/receive SH waves in plates and torsional waves in pipes. However, the efficiencies of these shear mode piezoelectric wafers were not compared yet. Thus, in this work, we conducted a comparative study on the performances of these three types of shear mode piezoelectric wafers in excitation and reception of SH waves in plates. Firstly, the definitions of these three types of shear mode piezoelectric wafers were presented. Then the wave patterns generated by different shear mode piezoelectric wafers were investigated by using finite element simulations. Finally, experiments were carried out to examine the efficiency of different shear mode piezoelectric wafers in $\mathrm{SH}_{0}$ wave generation and reception.

\section{Definition of Three Types Shear Modes in Piezoelectric Wafers}

When we talk about the shear mode in piezoelectric ceramics, we usually denote the conventional thickness-shear $\left(d_{15}\right)$ mode. That is, a conventional $d_{15}$ mode piezoelectric wafer is in-plane poled and the electric field is applied along the thickness direction, as shown in Figure 1a. Actually, for wafer-shaped piezoelectric ceramics used for guided wave generation/reception, there also exist another two shear modes, i.e., the thickness-poled thickness-shear $\left(\mathrm{d}_{15}\right)$ mode as shown in Figure $1 \mathrm{~b}$ and the in-plane poled face-shear $\left(\mathrm{d}_{24}\right)$ mode as shown in Figure 1c. From the material point of view, these three types of shear modes are equivalent since in all of them the electric field is applied perpendicularly to the poling direction. However, for wafer-shaped samples, they are different. Note that the thickness-poled $d_{15}$ mode and the face-shear $d_{24}$ mode have special advantages when used for SH wave excitation/reception [26,27].

(a)

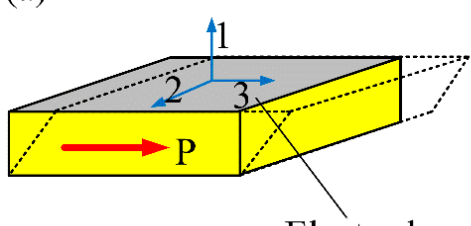

(b)

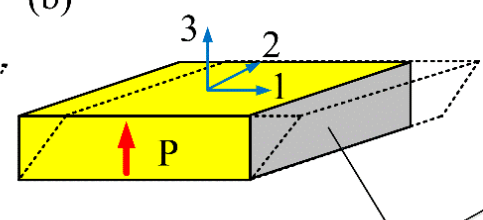

Electrode (c)

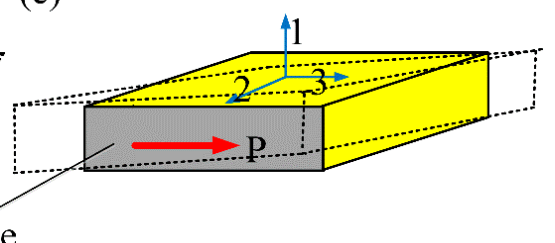

Figure 1. Definition of different shear mode piezoelectric wafers: (a) conventional in-plane poled thickness-shear $\mathrm{d}_{15}$ mode; (b) thickness-poled thickness-shear $\mathrm{d}_{15}$ mode; (c) face shear $\mathrm{d}_{24}$ mode.

In order for the convenience of analysis, Figure 2 presented the group velocity of guided waves for a $2 \mathrm{~mm}$-thick aluminum plate. It can be found that before the cut-off frequency, there only exist three wave modes, i.e., the $\mathrm{A}_{0}$ wave, $\mathrm{S}_{0}$ wave and $\mathrm{SH}_{0}$ wave. However, both the $\mathrm{S}_{0}$ wave and $\mathrm{A}_{0}$ wave 
are dispersive, which would lead signal distortion after wave propagation since the signal is excited in a certain bandwidth. In comparison, $\mathrm{SH}_{0}$ wave is totally non-dispersive.

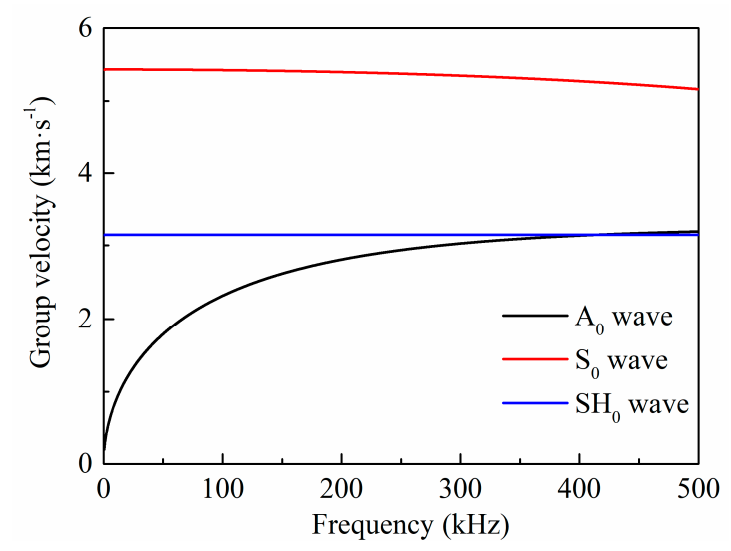

Figure 2. Group velocity verse frequency for $\mathrm{SH}_{0}$ wave and Lamb waves in a $2 \mathrm{~mm}$-thick aluminum plate.

\section{Finite Element Simulations}

Time-transient finite element (FEM) simulations were firstly performed using ANSYS to investigate the wave radiation patterns generated by different shear mode piezoelectric wafers. The dimensions of the three types shear mode piezoelectric wafers used here were all $8 \mathrm{~mm} \times$ $8 \mathrm{~mm} \times 1 \mathrm{~mm}$. The material was PZT-5H whose parameters can be found in [31].

The waveguide was selected to be a $400 \mathrm{~mm} \times 400 \mathrm{~mm} \times 2 \mathrm{~mm}$ aluminum plate with the PZT wafer bonded on the center of it. The largest size of elements is set to be less than $1 / 20$ the shortest wavelength, and the time step was set to be less than $1 / 20$ of the central frequency. Figure 3 presented the FEM simulated results of wave patterns generated by the three types shear mode piezoelectric wafers at $150 \mathrm{kHz}$ and $205 \mathrm{kHz}$. In cylindrical coordinates, the radial displacement component $u_{r}$, tangential displacement component $u_{\theta}$, and out-of-plane displacements component $u_{z}$ are dominated by the $\mathrm{S}_{0}$ wave, $\mathrm{SH}_{0}$ wave, and $\mathrm{A}_{0}$ wave, respectively [32]. Hence, we can verify that $u_{r}, u_{\theta}$, and $u_{z}$ were associated with the $\mathrm{S}_{0}$ wave, $\mathrm{SH}_{0}$ wave and $\mathrm{A}_{0}$ wave, respectively. It can be seen from Figure $3 \mathrm{a}, \mathrm{b}, \mathrm{d}$,e that both the in-plane poled and thickness-poled $\mathrm{d}_{15}$ piezoelectric wafers can generate $\mathrm{SH}_{0}$ wave along the bi-directions $\left(90^{\circ}\right.$ and $\left.270^{\circ}\right)$ perpendicular to both the poling direction and the field direction, and simultaneously generate Lamb waves along the orthogonal in-plane directions $\left(0^{\circ}\right.$ and $\left.180^{\circ}\right)$. At multiple frequencies, for both $\mathrm{d}_{15}$ mode wafers, the maximum amplitudes of the generated Lamb waves were always comparable to that of the $\mathrm{SH}_{0}$ wave. The decreased amplitude of the $\mathrm{A}_{0}$ wave at $150 \mathrm{kHz}$ was mainly caused by the effect of turning frequency [33]. Moreover, the radiation angle of the generated $\mathrm{A}_{0}$ wave is always significantly smaller than that of the generated $\mathrm{S}_{0}$ wave and $\mathrm{SH}_{0}$ wave. These results were in agreement with those reported in [21]. Note that the wave radiation patterns generated by using these two types $d_{15}$ piezoelectric wafers are exactly the same since they are both thickness shear modes.

In comparison, the generated wave pattern by the face shear $\mathrm{d}_{24}$ piezoelectric wafer was quite different, as shown in Figure $3 \mathrm{c}$,f. The $\mathrm{SH}_{0}$ wave was generated along four main directions $\left(0^{\circ}, 90^{\circ}\right.$, $180^{\circ}$ and $270^{\circ}$ ) with four-fold rotational symmetry. When deviating from the main directions, its amplitude decreased quickly and vanished at the wafer's diagonals $\left(45^{\circ}, 135^{\circ}, 225^{\circ}\right.$ and $\left.315^{\circ}\right)$ where Lamb waves including the $A_{0}$ wave and $S_{0}$ wave were generated with much smaller amplitudes. This phenomenon is not difficult to understand. Because of the pure face-shear deformation of the $d_{24}$ wafer, shear stress would be generated along the four sides of the wafer with the same amplitude, resulting in the generated four-fold rotational symmetric $\mathrm{SH}_{0}$ wave. Meanwhile, the shear stress would synthesize tensile/compressive stresses along the wafer's diagonals, leading to the generation of Lamb waves. 
It should be noted that at multiple frequencies, the wave field generated by the $\mathrm{d}_{24}$ wafer was always dominated by the $\mathrm{SH}_{0}$ wave. These results were in good agreement with those reported in [26].

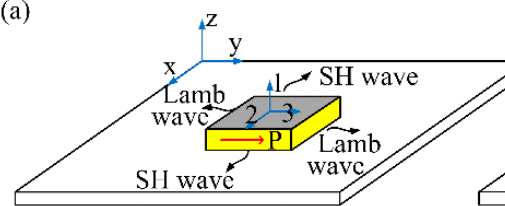

(d)

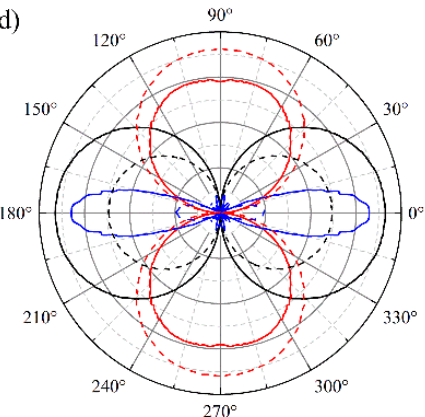

(b)

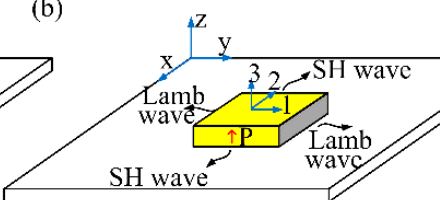

(e)

(c)
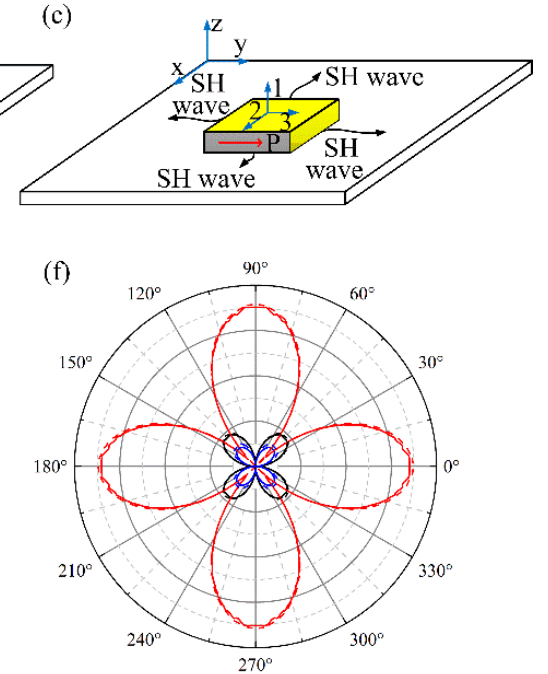

$150 \mathrm{kHz}:--u_{r}$ i.e. $\mathrm{S}_{0}$ wave $--u_{\theta}$ i.e. $\mathrm{SH}_{0}$ wave $--u_{z}$ i.e. $\mathrm{A}_{0}$ wave
$205 \mathrm{kHz}:-u_{r}$ i.e. $\mathrm{S}_{0}$ wave $-u_{\theta}$ i.e. $\mathrm{SH}_{0}$ wave $-u_{z}$ i.e. $\mathrm{A}_{0}$ wave

Figure 3. Illustration (up) and simulated results (bottom) of wave radiation patterns generated by using different shear modes piezoelectric wafers at $150 \mathrm{kHz}$ and $205 \mathrm{kHz}$. (a,d): in-plane poled $\mathrm{d}_{15}$ mode; $(\mathbf{b}, \mathbf{e})$ : thickness-poled $\mathrm{d}_{15}$ mode; $(\mathbf{c}, \mathbf{f})$ : face shear $\mathrm{d}_{24}$ mode.

\section{Experiments}

Experiments were then carried out to examine the efficiencies of different shear mode piezoelectric wafers in $\mathrm{SH}_{0}$ wave generation and reception. The experimental setup was shown in Figure 4.

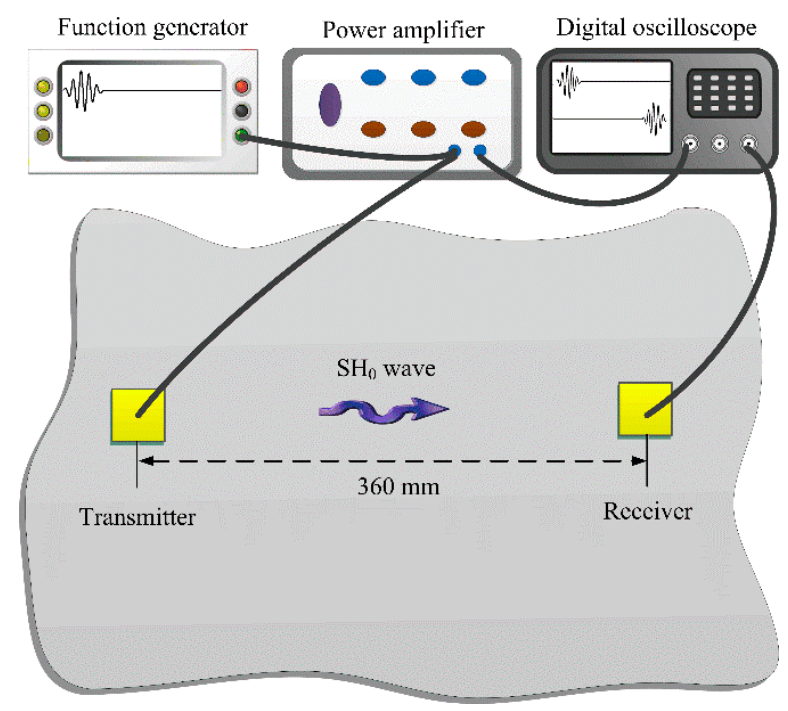

Figure 4. The schematic of experimental setup.

A $1000 \mathrm{~mm} \times 1000 \mathrm{~mm} \times 2 \mathrm{~mm}$ aluminum plate was used as the waveguide. The size of all three types of piezoelectric wafers was $8 \mathrm{~mm} \times 8 \mathrm{~mm} \times 1 \mathrm{~mm}$. The distance between the actuator and sensor was fixed at $360 \mathrm{~mm}$ so that the wave package can be separated in the time domain. When checking the wave generation performance, the different shear mode piezoelectric wafers served as actuators and 
the $\mathrm{d}_{36}$ type PMN-PT wafer $(5 \mathrm{~mm} \times 5 \mathrm{~mm} \times 1 \mathrm{~mm})$ was used as a sensor. Since the $\mathrm{d}_{36}$ type PMN-PT wafer can generate and receive both $\mathrm{SH}_{0}$ wave and Lamb waves, the wave velocity and purity can be examined at the same time [32]. When examining their performances in wave reception, the $d_{36}$ type PMN-PT wafer served as the actuator and the piezoelectric wafers were used as sensors. Finally, the same shear mode piezoelectric wafers were used as both actuator and sensor to further investigate its performance. During testing, a five-cycle sinusoid tone-burst modulated into the Hanning window was used as the exciting signal. The signal was generated by a function generator (3320A, Agilent, Palo Alto, CA, USA) and amplified by a power amplifier (Model 7602M, KROHN-HITE, Brockton, MA, USA). An oscilloscope (Agilent DSO-X 3024A) was used to record the signals received by the sensors with a tracing average of 128 times.

Before formal testing, the impedance of these three types shear mode piezoelectric wafers bonded on an aluminum plate were measured by using an impedance analyzer (Agilent 4294A), and the results from $90 \mathrm{kHz}$ to $300 \mathrm{kHz}$ were shown in Figure 5. It can be seen in Figure 5a that the impedances (including resistance and reactance) of the face-shear $\mathrm{d}_{24}$ mode wafer and the thickness-poled $\mathrm{d}_{15}$ mode were close to each other at most frequencies, and both are much larger than that of the conventional in-plane poled $d_{15}$ wafer. This is easy to understand because the in-plane poled $d_{15}$ wafer has larger electrodes and smaller distances between the electrodes. Therefore, it is expected that the normal drive voltage of the in-plane poled $d_{15}$ wafer should be lower than the other two. The non-monotonic of the impedance for the $d_{24}$ wafer near $200 \mathrm{kHz}$ was because there exists a resonance peak. Figure $5 \mathrm{~b}, \mathrm{c}$ presented the real image part i.e., resistance and imaginary part i.e., reactance of the impedance respectively. As shown in Figure $5 b$, for both two types $d_{15}$ wafer, the resistance decreased firstly and then increased slightly with the increasing frequency. However, the changes in amplitude for the in-plane poled $d_{15}$ wafer was much smaller than that of thickness-poled $d_{15}$ wafer. In comparison, the resistance of the $\mathrm{d}_{24}$ wafer increased firstly and then decreased quickly with the increasing frequency. The inflection point of its curves was near its resonance frequency i.e., $200 \mathrm{kHz}$. Regarding the reactance shown in Figure $5 c$, for both two types $d_{15}$ wafer, it decreased continuously with frequency increasing. One thing should be noted that for both two types $d_{15}$ wafer, the amplitudes' change in reactance was much larger than that of resistance, resulting in continue decreasing of their impedance with the increasing frequency, as shown in Figure $5 \mathrm{a}$. For the reactance of the $\mathrm{d}_{24}$ wafer, there appeared slight increase in a small frequency range near $200 \mathrm{kHz}$. The changes in the resistance and reactance for the $\mathrm{d}_{24}$ wafer near $200 \mathrm{kHz}$ codetermined its non-monotonic in impedance. It should be noted that the impedance of the wafers was dependent on their size, material and deformation mode.
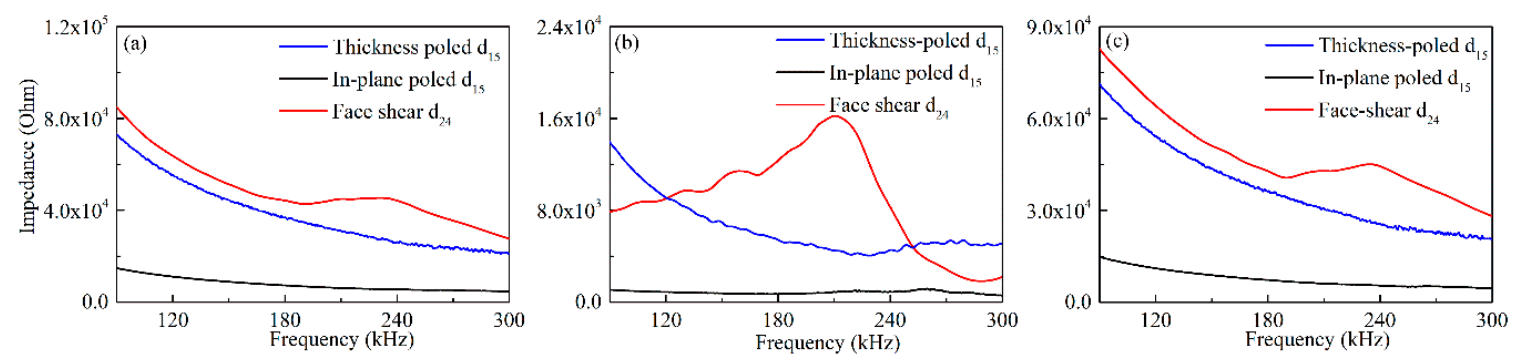

Figure 5. The impedance spectrum of three types shear mode piezoelectric wafers with the same dimensions of $8 \mathrm{~mm} \times 8 \mathrm{~mm} \times 1 \mathrm{~mm}$ bonded on the aluminum plate: (a) impedance; (b) resistance; (c) reactance.

\subsection{Performance of Different Shear Mode Piezoelectric Wafers in SH Wave Generation}

Firstly, the performance of different shear mode piezoelectric wafers in $\mathrm{SH}_{0}$ wave generation was compared. The signals were generated by different shear mode wafers and received by the $d_{36}$ type PMN-PT wafer. For all three types of wafer of $8 \mathrm{~mm} \times 8 \mathrm{~mm} \times 1 \mathrm{~mm}$ size, according to our previous work, the $\mathrm{SH}_{0}$ wave can be effectively generated from $90 \mathrm{kHz}$ to $270 \mathrm{kHz}$ in the $2 \mathrm{~mm}$-thick aluminum 
plate. Hence the testing frequency was set in this range. During experiments, all wafers were excited by using a fixed voltage of $20 \mathrm{~V}$ firstly and then using a fixed power consumption of $0.08 \mathrm{~W}$.

In order to verify the wave velocity generated by the different types wafers, the continuous wavelet transform (CWT) was adopted to analyze the signals. Due to the space limitations, only the wave generated by the $d_{24}$ wafer with drive voltage of $20 \mathrm{~V}$ at $200 \mathrm{kHz}$ was presented as an example. It can be seen in Figure 6a that only one wave package appeared in the received signal. After applying the CWT to the signals, the time internal between the drive signal and the received signal was recognized to be $116.8 \mu \mathrm{s}$, as shown in Figure $6 \mathrm{~b}$. The corresponding group wave velocity was calculated to be $3082 \mathrm{~mm} \cdot \mathrm{s}^{-1}$, which was agreement with the theoretical value of $\mathrm{SH}_{0}$ wave in an aluminum plate, i.e., $3099 \mathrm{~mm} \cdot \mathrm{s}^{-1}$. The group velocity of the waves generated by different shear mode piezoelectric wafers verses frequency was plotted in Figure 6c. It can be found that within the testing frequency range, the experimental results for all three types wafers was agreement with theoretical value of $\mathrm{SH}_{0}$ wave within error less than $2.5 \%$. Figure 7 presents the results at $150 \mathrm{kHz}$ and $200 \mathrm{kHz}$, respectively for all the three wafers with drive voltage of $20 \mathrm{~V}$, from which it can be found that the $\mathrm{SH}_{0}$ wave can be generated successfully for all three types of wafers.
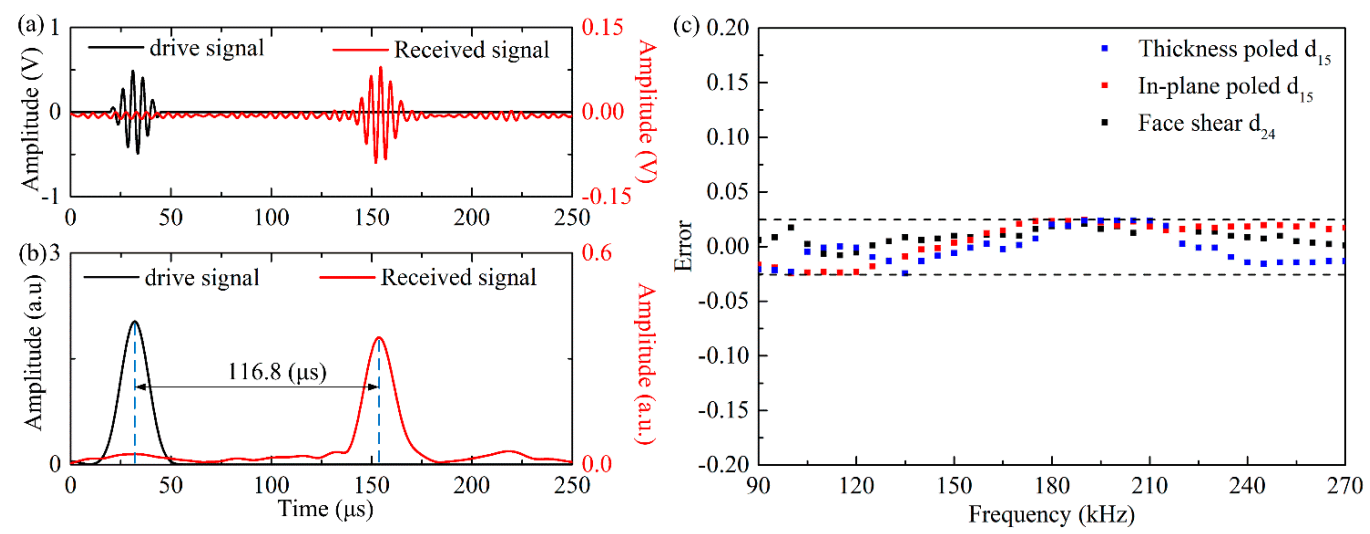

Figure 6. (a) Wave signals generated by the $d_{24}$ piezoelectric wafer and received by the $d_{36}$ type PMN-PT wafer with drive voltage of $20 \mathrm{~V}$ at $200 \mathrm{kHz}$; (b) continue wavelet transform (CWT) of the drive signal and receive signal in (a); (c) Frequency dependent relative errors of the measured group velocity of the $\mathrm{SH}_{0}$ waves generated by different shear mode piezoelectric wafers.

The amplitude of the $\mathrm{SH}_{0}$ wave versus frequency was plotted in Figure 8. It can be seen from Figure $8 \mathrm{a}$ that for all the three types wafers, with the increasing frequency, the amplitudes of the generated $\mathrm{SH}_{0}$ wave increase first and then decrease gradually under the drive voltage of $20 \mathrm{~V}$. Furthermore, the $\mathrm{SH}_{0}$ wave amplitude generated by the in-plane poled $\mathrm{d}_{15}$ wafer is always the maximum and that by the thickness-poled $\mathrm{d}_{15}$ wafer is always the minimum. The $\mathrm{SH}_{0}$ wave amplitude generated by the face-shear $d_{24}$ wafer is always between the former two. Then, the three types shear mode wafers were excited by fixing the power consumption of $0.08 \mathrm{~W}$, and the corresponding drive voltage for each wafer at different frequencies was calculated based on the Ohm's law using the measured impedance spectrum in Figure 5. The obtained $\mathrm{SH}_{0}$ wave amplitude versus frequency was plotted in Figure $8 \mathbf{b}$, which is quite different from Figure $8 \mathbf{a}$. The thickness-poled $d_{15}$ wafer still generates the minimum amplitude of $\mathrm{SH}_{0}$ wave. The face-shear $\mathrm{d}_{24}$ wafer generates $\mathrm{SH}_{0}$ wave with the same amplitude as that by the in-plane poled $\mathrm{d}_{15}$ wafer below $130 \mathrm{kHz}$. While above $130 \mathrm{kHz}$, the $\mathrm{SH}_{0}$ wave amplitude generated by the $\mathrm{d}_{24}$ wafer increased much quickly than that by the in-plane poled $d_{15}$ wafer. These results indicated that the energy conversion efficiency of the face shear $d_{24}$ wafer is better than that of the in-plane poled $d_{15}$ wafer, and much better than that of the thickness-poled $\mathrm{d}_{15}$ wafer. 

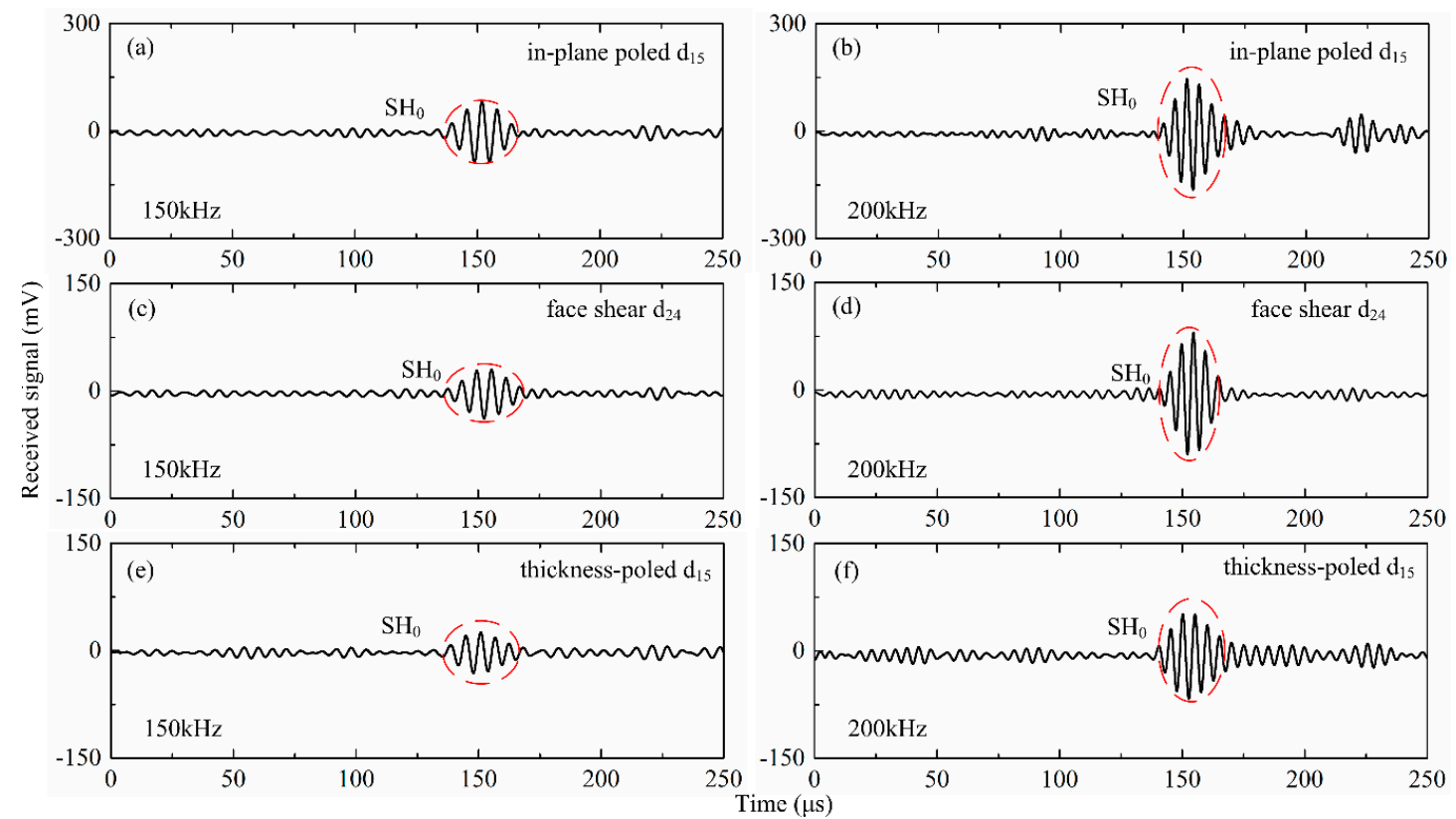

Figure 7. Wave signals generated by different shear mode piezoelectric wafers and received by the $d_{36}$ type PMN-PT wafer with drive voltage of $20 \mathrm{~V}$ at $150 \mathrm{kHz}$ (left) and $200 \mathrm{kHz}$ (right). (a,b): in-plane poled $\mathrm{d}_{15}$ mode; $(\mathbf{c}, \mathbf{d})$ : face shear $\mathrm{d}_{24}$ mode; $(\mathbf{e}, \mathbf{f})$ : thickness-poled $\mathrm{d}_{15}$ mode.
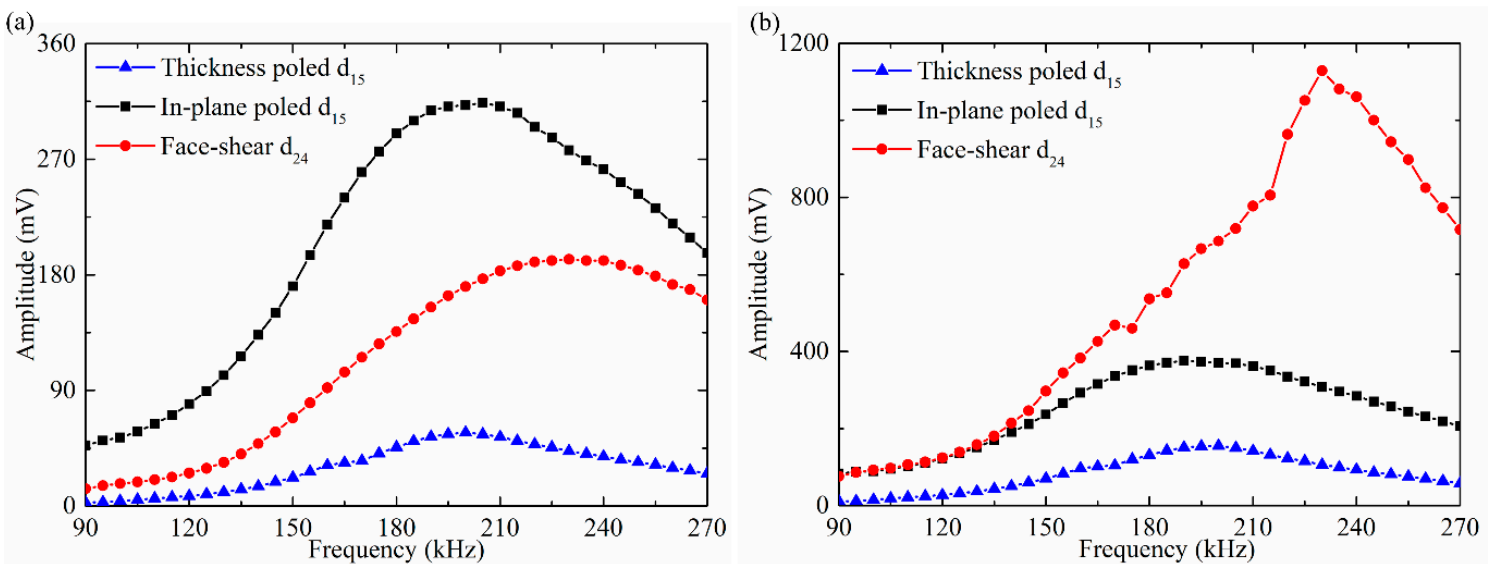

Figure 8. The amplitude of generated $\mathrm{SH}_{0}$ wave versus frequency. Signals were generated by different shear mode piezoelectric wafers and received by the $d_{36}$ type PMN-PT wafer under (a) fixed drive voltage of $20 \mathrm{~V}$ and (b) fixed power consumption of $0.08 \mathrm{~W}$.

The testing results in Figure 8 can be explained as follows. When fixing the drive voltage, the energy consumption by the in-plane poled $d_{15}$ wafer is the maximum because its impedance is the minimum, thus it always generated the maximum $\mathrm{SH}_{0}$ wave amplitude, as shown in Figure $8 \mathrm{a}$. The other factor that can affect the generated wave amplitude is the deformation mechanism of the wafers. For the thickness shear $\mathrm{d}_{15}$ mode (both in-plane poled and thickness-poled), its deformation was actually the simple shear which was not self-balancing, which means the deformation cannot be transferred from the wafer to the waveguide in quasi-static case and can only be transferred in dynamic case via the inertial effect. This is why some researchers believe that the external constraint was needed for the thickness shear $d_{15}$ wafers [34]. Figure $8 b$ also showed that the wave driving efficiency of the in-plane poled $d_{15}$ wafer was actually acceptable, even without any external constraint. 
In comparison, the face-shear deformation mode is self-balancing. Thus, the deformation could effectively be transferred to the hosting structure even in the quasi-static case without the inertial effect. Hence, when fixing the power consumption, the amplitude of $\mathrm{SH}_{0}$ wave generated by the face shear $\mathrm{d}_{24}$ wafer was maximum, as plotted in Figure $8 \mathrm{~b}$. The lowest amplitude of $\mathrm{SH}_{0}$ wave generated by the thickness-poled $d_{15}$ wafer in Figure $8 b$ indicated that although the thickness-poled $d_{15}$ mode was equivalent to the in-plane poled $\mathrm{d}_{15}$ mode in deformation, its deformation which could propagate from the transducer to the waveguide was considerably lower.

\subsection{Performances of Different Shear Mode Piezoelectric Wafers in SH Wave Reception}

Next the performance of different shear mode piezoelectric wafers in $\mathrm{SH}_{0}$ wave reception was compared. The signals were generated by the $\mathrm{d}_{36}$ type PMN-PT wafer under $20 \mathrm{~V}$ and received by different shear mode piezoelectric wafers. The testing results were shown in Figure 9. It can be seen that within the testing frequency from $90 \mathrm{kHz}$ to $270 \mathrm{kHz}$, the $\mathrm{SH}_{0}$ wave amplitude received by the face shear $\mathrm{d}_{24}$ wafer was always larger than that by the other two wafers. Above $160 \mathrm{kHz}$, the $\mathrm{SH}_{0}$ wave amplitude received by the face shear $\mathrm{d}_{24}$ wafer was about three times of that received by the in-plane poled $d_{15}$ wafer and more than four times of that received by the thickness-poled $d_{15}$ wafer. The excellent reception performance of the face-shear $d_{24}$ wafer should be attributed to its in-plane shear deformation mechanism which is self-balancing. This result indicated that the face shear $d_{24}$ wafer was much more suitable to act as an $\mathrm{SH}$ wave sensor than both types thickness shear $\mathrm{d}_{15}$ wafers.

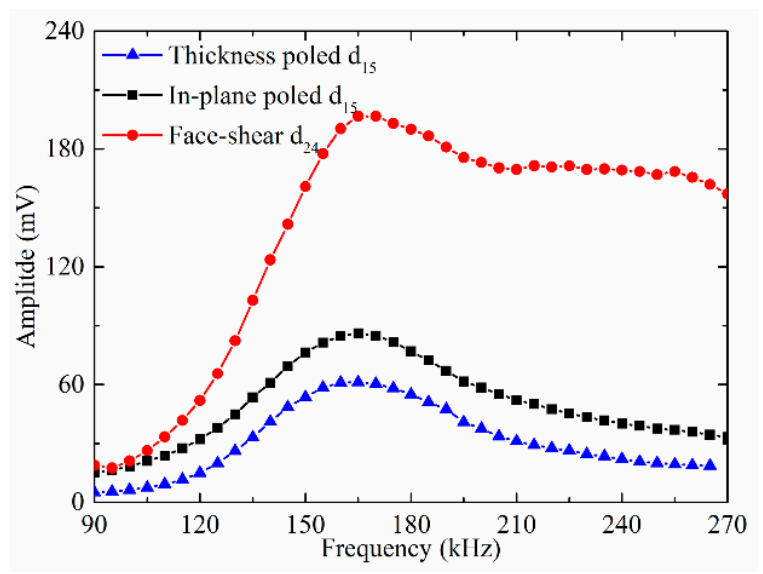

Figure 9. The amplitude of received $\mathrm{SH}_{0}$ wave versus frequency. Signals were generated by the $\mathrm{d}_{36}$ type PMN-PT wafer under $20 \mathrm{~V}$ and received by different shear mode piezoelectric wafers.

\subsection{Signals Generated and Received by the Same Shear Mode Piezoelectric Wafers}

In practical applications, wave signals are usually generated and received by the same type of transducers. Hence it is necessary to test wave signals generated and received by the same shear mode piezoelectric wafers. Figure 10a presented the results with the drive voltage of $20 \mathrm{~V}$. It can be seen that in the whole testing frequency range from $90 \mathrm{kHz}$ to $270 \mathrm{kHz}$, the $\mathrm{SH}_{0}$ wave amplitude generated and received by the thickness-poled $d_{15}$ wafer was always the minimum. When the operating frequency was below $175 \mathrm{kHz}$, the $\mathrm{SH}_{0}$ wave amplitude generated and received by the in-plane poled $\mathrm{d}_{15}$ wafers was larger than that by the face shear $d_{24}$ wafer. While above $175 \mathrm{kHz}$, the tendency was totally reversed. 
(a)

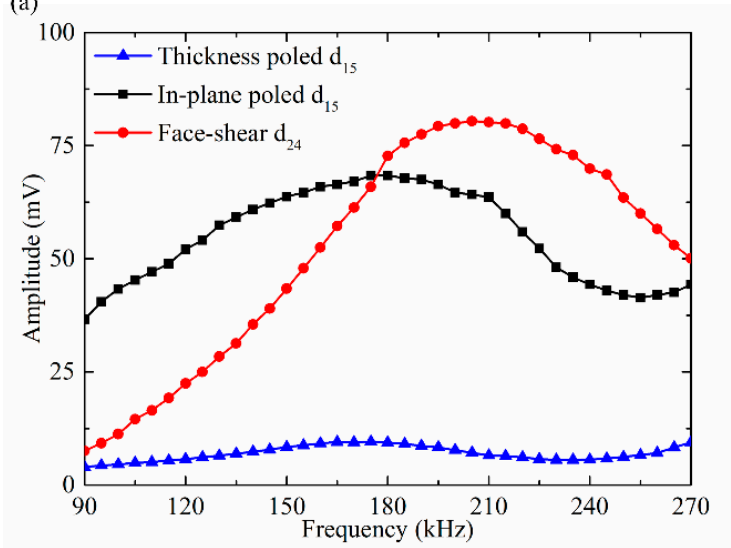

(b)

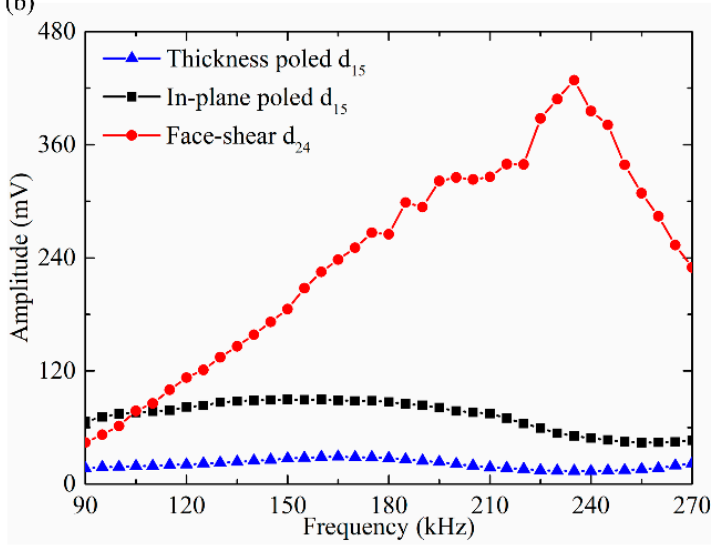

Figure 10. The $\mathrm{SH}_{0}$ wave amplitude versus frequency. Signals were generated and received by the same type shear mode piezoelectric wafer under (a) fixed drive voltage of $20 \mathrm{~V}$, (b) fixed power consumption of $0.08 \mathrm{~W}$.

When the PZT wafers were excited under the same power consumption, the results were quite different. As plotted in Figure 10b where all wafers were excited with the same power consumption of $0.08 \mathrm{~W}$, the $\mathrm{SH}_{0}$ wave amplitude generated and received by the face shear $\mathrm{d}_{24}$ wafers was the maximum above $110 \mathrm{kHz}$. In most frequency ranges, its amplitude was over five times greater than that by the in-plane poled $d_{15}$ wafer and more than ten times greater than that by the thickness-poled $d_{15}$ wafer. These results were also in agreement with that plotted in Figures 8 and 9 . From these results, it can be inferred that the in-plane poled $\mathrm{d}_{15}$ wafer was more suitable to act as a $\mathrm{SH}$ wave transducer at low frequencies (below $175 \mathrm{kHz}$ ) but with large power consumption, while the face shear $\mathrm{d}_{24}$ wafer was more suitable to act as a $\mathrm{SH}$ wave transducer with high energy conversion efficiency within all the testing frequencies.

In practical applications, besides the wave amplitude, the signal to noise ratio (SNR) is another important parameter to evaluate a transducer. Thus, the $\mathrm{SNR}$ of $\mathrm{SH}_{0}$ wave generated and received by the same shear mode piezoelectric wafer with drive voltage of $20 \mathrm{~V}$ was plotted in Figure 11a. It can be found that the $\mathrm{SNR}$ of the $\mathrm{SH}_{0}$ wave generated/received by the face shear $\mathrm{d}_{24}$ wafer was more than $20 \mathrm{~dB}$ above $130 \mathrm{kHz}$, and can even reach $26 \mathrm{~dB}$ around $200 \mathrm{kHz}$. In comparison, the SNR of the $\mathrm{SH}_{0}$ wave by the in-plane poled $\mathrm{d}_{15}$ wafer was almost constant at around $16 \mathrm{~dB}$ above $110 \mathrm{kHz}$. The SNR of the $\mathrm{SH}_{0}$ wave by the thickness-poled $\mathrm{d}_{15}$ wafer was very small in most frequencies, while in a narrow band from $150 \mathrm{kHz}$ to $180 \mathrm{kHz}$, it is slightly better than that by the in-plane poled $\mathrm{d}_{15}$ wafer, i.e., above $16 \mathrm{~dB}$.

To explain this, the wave signals generated by different types of wafers at $165 \mathrm{kHz}$ were plotted in Figure $11 b-d$. It can be seen that the SNR for the face shear $d_{24}$ wafer was maximum and that for the in-plane poled $d_{15}$ wafer is minimum, which is in agreement with that in Figure 11a. Although the $\mathrm{SH}_{0}$ wave amplitude generated by the thickness-poled $\mathrm{d}_{15}$ wafer was much lower than that by the in-plane $d_{15}$ wafer, its SNR was still slightly higher because of the much lower noise. It should be noted that the $\mathrm{SNR}$ of different types of wafers in $\mathrm{SH}_{0}$ wave generation is almost independent of the drive voltage. From the results in Figure 11, it can be concluded that the face shear $\mathrm{d}_{24}$ wafer was considerably better than the in-plane poled $\mathrm{d}_{15}$ wafer and much better than the thickness-poled $\mathrm{d}_{15}$ wafer in $\mathrm{SH}_{0}$ wave generation and reception. 

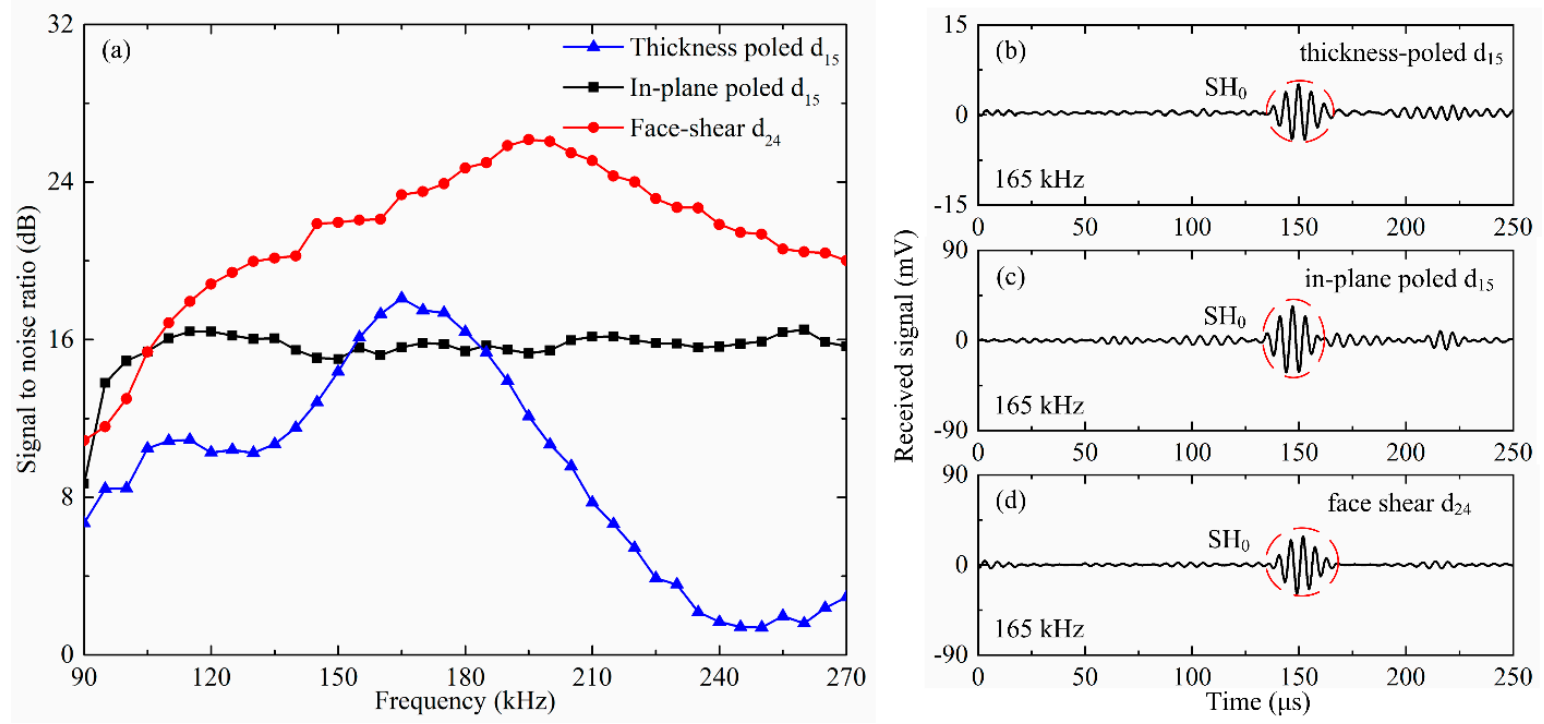

Figure 11. The signal to noise ratio (SNR) of $\mathrm{SH}_{0}$ wave generated and received by the same type shear mode piezoelectric wafers. (a) SNR verses frequency. Wave signals generated by (b) in-plane poled $d_{15}$ wafer; (c) face shear $d_{24}$ mode wafer and (d) thickness-poled $d_{15}$ mode wafer under the drive voltage of $20 \mathrm{~V}$ at $165 \mathrm{kHz}$.

Finally, in order to compare the performances of different shear mode piezoelectric wafers in a more intuitive manner, all the results presented above were summarized and listed in Table 1. It can be clearly seen that overall the face shear $d_{24}$ wafer was considerably superior to the in-plane poled $d_{15}$ wafers and much better than the thickness-poled $d_{15}$ wafer when used as SH wave transducers.

Table 1. The performances of different shear mode piezoelectric wafers in $\mathrm{SH}_{0}$ wave generation and reception.

\begin{tabular}{|c|c|c|c|c|c|c|}
\hline \multirow[t]{2}{*}{ Modes } & \multicolumn{2}{|c|}{ Amplitude in Generation } & \multirow{2}{*}{$\begin{array}{l}\text { Amplitude in } \\
\text { Reception }\end{array}$} & \multicolumn{2}{|c|}{$\begin{array}{c}\text { Amplitude in } \\
\text { Self-Generation/Reception }\end{array}$} & \multirow{2}{*}{$\begin{array}{c}\text { Signal to Noise Ratio } \\
\text { (SNR) } \\
\text { Fixed Voltage }\end{array}$} \\
\hline & $\begin{array}{l}\text { Fixed } \\
\text { Voltage }\end{array}$ & $\begin{array}{l}\text { Fixed Power } \\
\text { Consumption }\end{array}$ & & Fixed Voltage & $\begin{array}{l}\text { Fixed Power } \\
\text { Consumption }\end{array}$ & \\
\hline In-plane poled $d_{15}$ & high & moderate & moderate & $\begin{array}{l}\text { High below } 175 \mathrm{kHz} \\
\text { moderate above } 175 \mathrm{kHz}\end{array}$ & moderate & $\sim 16 \mathrm{~dB}$ \\
\hline Face shear $d_{24}$ & moderate & high & high & $\begin{array}{c}\text { moderate below } 175 \mathrm{kHz} \\
\text { High above } 175 \mathrm{kHz}\end{array}$ & high & typically above $20 \mathrm{~dB}$ \\
\hline Thickness-poled $d_{15}$ & low & low & low & low & low & typically below $12 \mathrm{~dB}$ \\
\hline
\end{tabular}

\section{Conclusions}

In summary, we presented a systematic comparison of three types of shear mode piezoelectric wafers in SH wave generation and reception. The results indicated the face shear $\mathrm{d}_{24}$ wafer can generate almost single mode $\mathrm{SH}$ wave while the in-plane poled $\mathrm{d}_{15}$ wafer and the thickness-poled $\mathrm{d}_{15}$ wafer will generate both Lamb waves and SH wave. When served as an actuator, the in-plane poled $\mathrm{d}_{15}$ wafer can generate $\mathrm{SH}_{0}$ wave in high amplitude but with large power consumption, while the face shear $\mathrm{d}_{24}$ wafer can generate $\mathrm{SH}_{0}$ wave with high energy conversion efficiency. When served as a sensor, the face shear $d_{24}$ wafer was obviously superior to the other two types of thickness shear $d_{15}$ wafers. Besides, in most testing frequencies, the signal to noise ratio of the face shear $\mathrm{d}_{24}$ wafer was also the best in self-generation/reception. It should be mentioned that although the performances of the thickness-poled $\mathrm{d}_{15}$ wafer was not very good in $\mathrm{SH}_{0}$ wave generation and reception, it is very suitable for omni-directional SH wave piezoelectric transducers because of its unique configuration [27]. This work may provide useful guidance for SHM-based SH wave generation/reception. 
Author Contributions: Conceptualization, F.L.; Methodology, F.L. and Q.H.; Software, Q.H.; Validation, Q.H. and M.C.; Formal Analysis, Q.H., M.C. and F.L.; Investigation, Q.H. and M.C.; Resources, Q.H. and M.C.; Data Curation, Q.H.; Writing-Original Draft Preparation, Q.H.; Writing-Review \& Editing, Q.H., M.C. and F.L.; Visualization, Q.H. and M.C.; Supervision, F.L.; Project Administration, F.L.; Funding Acquisition, F.L.

Funding: This work is supported by the National Natural Science Foundation of China under Grant No.11672003 and by the National Key Research and Development Program under Grant No. 2016YFF0203002.

Conflicts of Interest: The authors declare no conflict of interest.

\section{References}

1. Chang, F.K. Structural Health Monitoring 2000; CRC Press: Boca Raton, FL, USA, 1999.

2. Balageas, D.; Fritzen, C.P.; Güemes, A. Structural Health Monitoring; John Wiley \& Sons: Hoboken, NY, USA, 2010.

3. Raghavan, A.; Cesnik, C.E. Review of Guided-Wave Structural Health Monitoring. Available online: http:// pdfs.semanticscholar.org/b29f/c5dafcc2108693e985c0d69b3c99c3d40c62.pdf (accessed on 14 August 2018).

4. Croxford, A.J.; Wilcox, P.D.; Drinkwater, B.W.; Konstantinidis, G. Strategies for guided-wave structural health monitoring. Proc. R. Soc. A 2007, 463, 2961-2981. [CrossRef]

5. Giurgiutiu, V. Structural Health Monitoring with Piezoelectric Wafer Active Sensors; Elsevier: Amsterdam, The Netherlands, 2007.

6. Alleyne, D.N.; Cawley, P. Optimization of Lamb wave inspection techniques. NDT E Int. 1992, 25, 11-22. [CrossRef]

7. Kessler, S.S.; Spearing, S.M.; Soutis, C. Damage detection in composite materials using Lamb wave methods. Smart Mater. Struct. 2002, 11, 269. [CrossRef]

8. Ebrahimkhanlou, A.; Salamone, S. Single-Sensor Acoustic Emission Source Localization in Plate-Like Structures Using Deep Learning. Aerospace 2018, 5, 50. [CrossRef]

9. Ebrahimkhanlou, A.; Salamone, S. A probabilistic framework for single-sensor acoustic emission source localization in thin metallic plates. Smart Mater. Struct. 2017, 26, 095026. [CrossRef]

10. Ebrahimkhanlou, A.; Dubuc, B.; Salamone, S. Damage localization in metallic plate structures using edge-reflected lamb waves. Smart Mater. Struct. 2016, 25, 085035. [CrossRef]

11. Kundu, T. Acoustic source localization. Ultrasonics 2014, 54, 25-38. [CrossRef] [PubMed]

12. Kabir, M.; Kazari, H.; Ozevin, D. Piezoelectric MEMS Acoustic Emission Sensors. Sens. Actuators A Phys. 2018, 279, 53-64. [CrossRef]

13. Park, H.W.; Kim, S.B.; Sohn, H. Understanding a time reversal process in Lamb wave propagation. Wave Motion 2009, 46, 451-467. [CrossRef]

14. Su, Z.; Ye, L. Selective generation of Lamb wave modes and their propagation characteristics in defective composite laminates. Proc. Inst. Mech. Eng. 2004, 218, 95-110. [CrossRef]

15. Nakamura, N.; Ogi, H.; Hirao, M.; Nakahata, K. Mode conversion behavior of SH guided wave in a tapered plate. NDT E Int. 2012, 45, 156-161.

16. Petcher, P.; Burrows, S.; Dixon, S. Shear horizontal (SH) ultrasound wave propagation around smooth corners. Ultrasonics 2014, 54, 997-1004. [CrossRef] [PubMed]

17. Martin, S.; Ricco, A.; Niemczyk, T.; Frye, G. Characterization of SH acoustic plate mode liquid. Sens. Actuators 1989, 20, 253-268. [CrossRef]

18. Thompson, R.B. Generation of horizontally polarized shear waves in ferromagnetic materials using magnetostrictively coupled meander-coil electromagnetic transducers. Appl. Phys. Lett. 1979, 34, 175-177. [CrossRef]

19. Vasile, C.; Thompson, R. Excitation of horizontally polarized shear elastic waves by electromagnetic transducers with periodic permanent magnets. J. Appl. Phys. 1979, 50, 2583-2588. [CrossRef]

20. Wilcox, P.; Lowe, M.; Cawley, P. Lamb and SH wave transducer arrays for the inspection of large areas of thick plates. In Proceedings of the AIP Conference, Montreal, QC, Canada, 25-30 July 1999.

21. Kamal, A.; Giurgiutiu, V. Shear horizontal wave excitation and reception with shear horizontal piezoelectric wafer active sensor (SH-PWAS). Smart Mater. Struct. 2014, 23, 085019. [CrossRef]

22. Alleyne, D.; Cawley, P. The excitation of Lamb waves in pipes using dry-coupled piezoelectric transducers. J. Nondestr. Eval. 1996, 15, 11-20. [CrossRef] 
23. Alleyne, D.N.; Pavlakovic, B.; Lowe, M.J.S.; Cawley, P. Rapid, long range inspection of chemical plant pipework using guided waves. In Proceedings of the AIP Conference, Ames, IA, USA, 16-20 July 2000.

24. Liu, Z.; He, C.; Wu, B.; Wang, X.; Yang, S. Circumferential and longitudinal defect detection using T $(0,1)$ mode excited by thickness shear mode piezoelectric elements. Ultrasonics 2006, 44, 1135-1138. [CrossRef] [PubMed]

25. Belanger, P.; Boivin, G. Development of a low frequency omnidirectional piezoelectric shear horizontal wave transducer. Smart Mater. Struct. 2016, 25, 045024. [CrossRef]

26. Miao, H.; Huan, Q.; Li, F. Excitation and reception of pure shear horizontal waves by using face-shear d24 mode piezoelectric wafers. Smart Mater. Struct. 2016, 25, 11LT01. [CrossRef]

27. Huan, Q.; Miao, H.; Li, F. A uniform-sensitivity omnidirectional shear-horizontal (SH) wave transducer based on a thickness poled, thickness-shear (d15) piezoelectric ring. Smart Mater. Struct. 2017, 26, 08 LT01. [CrossRef]

28. Huan, Q.; Miao, H.; Li, F. A variable-frequency structural health monitoring system based on omnidirectional shear horizontal wave piezoelectric transducers. Smart Mater. Struct. 2018, 27, 025008. [CrossRef]

29. Miao, H.; Huan, Q.; Wang, Q.; Li, F. Excitation and reception of single torsional wave T $(0,1)$ mode in pipes using face-shear d24 piezoelectric ring array. Smart Mater. Struct. 2017, 26, 025021. [CrossRef]

30. Miao, H.; Huan, Q.; Wang, Q.; Li, F. A new omnidirectional shear horizontal wave transducer using face-shear (d 24) piezoelectric ring array. Ultrasonics 2017, 74, 167-173. [CrossRef] [PubMed]

31. Miao, H.; Dong, S.; Li, F. Excitation of fundamental shear horizontal wave by using face-shear (d36) piezoelectric ceramics. J. Appl. Phys. 2016, 119, 174101. [CrossRef]

32. Zhou, W.; Li, H.; Yuan, F.G. Fundamental understanding of wave generation and reception using d 36 type piezoelectric transducers. Ultrasonics 2015, 57, 135-143. [CrossRef] [PubMed]

33. Giurgiutiu, V. Tuned Lamb wave excitation and detection with piezoelectric wafer active sensors for structural health monitoring. J. Intell. Mater. Syst. Struct. 2005, 16, 291-305. [CrossRef]

34. Köhler, B.; Gaul, T.; Lieske, U.; Schubert, F. Shear horizontal piezoelectric fiber patch transducers (SH-PFP) for guided elastic wave applications. NDT E Int. 2016, 82, 1-12. [CrossRef]

(C) 2018 by the authors. Licensee MDPI, Basel, Switzerland. This article is an open access article distributed under the terms and conditions of the Creative Commons Attribution (CC BY) license (http:/ / creativecommons.org/licenses/by/4.0/). 